\title{
Aspirin therapy in the primary prevention of coronary heart disease and stroke - yes or no
}

\section{(D) Vjeran Nikolić Heitzler ${ }^{\star}$}

The Polyclinic Drinković, Zagreb, Croatia

\section{RECEIVED:}

November 28, 2020

ACCEPTED:

December 18, 2020

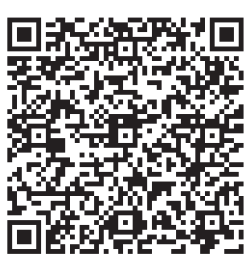

KEYWORDS: aspirin, primary prevention, atherosclerotic cardiovascular disease.
CITATION: Cardiol Croat. 2021;16(1-2):68. | https://doi.org/10.15836/ccar2021.68

*ADDRESS FOR CORRESPONDENCE: Vjeran Nikolić Heitzler, Poliklinika "Ivo Drinković", Zagreb, Šulekova 5, HR10000 Zagreb, Croatia. / Phone: +385-98-385-002 / E-mail: vjeran.nikolicheitzler@gmail.com

ORCID: Vjeran Nikolić Heitzler, https://orcid.org/0000-0001-5332-391X

The goal: In everyday practice, we doctor often prescribe aspirin. The question arises whether this is always justified. It is necessary to discern the use of aspirin in primary prevention in people with no signs of cardiovascular disease from use in secondary prevention in at-risk individuals with previous heart attacks and strokes or known cardiovascular diseases (CVD). Coronary heart disease and stroke, the principal manifestations of CVD, are the first and second most common causes of death worldwide. The World Health Organization predicts that, by 2020, coronary heart disease will become the world's most important cause of death and disability and, further, the most important cause of premature death, "if the victims of the COVID-19 pandemic do not overtake them" (authors comment). Low doses of aspirin were considered indispensable in the prevention of heart attack and stroke and other CVD. New guidelines, though, suggest that aspirin should not be prescribed to most adults who are in good cardiovascular health and that the risk of internal bleeding often outweighs the benefit.

According to the European Guidelines for Cardiovascular Diseases and Prevention in 2016, using the experience of older historical trials, aspirin is recommended for the primary prevention of people with high CVD risk (especially those with a ten-year mortality risk of over 10\%). The Antiplatelet Trialists Collaboration meta-analysis demonstrated the benefits of antithrombotic therapy (mainly aspirin) in patients with type 1 or type 2 DM with clinically established CAD, cerebrovascular disease or other forms of thrombotic disease, with a $25 \%$ reduction in risk of CV events. ${ }^{1}$

The use of aspirin for the primary prevention of CVD has been a subject of intense debate since the publication of three large, randomized clinical trials in 2018 (ASCEND, ARRIVE, and ASPREE). The American College of Cardiology and American Heart Association released the new guidelines in 2019 and one of the mayor changes is recommendation against the broad use of aspirin in primary prevention. ${ }^{2}$ Low-dose aspirin 100 milligrams or less did not help older adults who do not have CVD. The new trials published 2019, meta-analysis of aspirin effects in primary prevention of CVD comprising 13 randomized-controlled trials in 164.225 patents comparing aspirin versus placebo/control during a mean follow-up period of 6.4 years suggested that the benefit of aspirin for primary prevention has been neutralized, perhaps due to the use of other modern preventive therapies, such as statins, and general improvements in the population prevalence of smoking and hypertension. The authors of the new guidelines said low-dose aspirin should not be routinely given as a preventive measure to adults 70 years and older or to any adult who has an increased risk of bleeding. Aspirin did reduce CVD by a modest $11 \%$, with a number needed to treat to prevent 1 event of 265 , but it also increased major bleeding, such as serious GI bleed, intracranial bleed or bleed needing hospitalization or transfusion by $43 \%$. That is a number needed to harm of 210. Among major bleeding events, intracranial hemorrhage is associated with high mortality rates and functional dependency. ${ }^{3}$

Conclusion: Aspirin remains an important medication for acute management of vascular events; for use after certain procedures; for secondary prevention; and, only after careful selection of the right patients, for primary prevention.

LITERATURE IIIIIIIIIIIIIIIIIIIIIIIIIIIIIIIIIIIIIIIIIIIIIIIIIIIIIIIIIIIIIIIIIIIIIIIIIIIIIIIIIIIIIIIIIIIIIIIII

1. Collaborative overview of randomised trials of antiplatelet therapy--I: Prevention of death, myocardial infarction, and stroke by prolonged antiplatelet therapy in various categories of patients. Antiplatelet Trialists' Collaboration. BMJ. 1994 Jan 8;308(6921):81-106. https://doi.org/10.1136/bmj.308.6921.81

2. Gelbenegger G, Postula M, Pecen L, Halvorsen S, Lesiak M, Schoergenhofer C, et al. Aspirin for primary prevention of cardiovascular disease: a meta-analysis with a particular focus on subgroups. BMC Med. 2019 Nov 4;17(1):198. https://doi.org/10.1186/12916-019-1428-0

3. Huang WY, Saver JL, Wu YL, Lin CJ, Lee M, Ovbiagele B. Frequency of Intracranial Hemorrhage With Low-Dose Aspirin in Individuals Without Symptomatic Cardiovascular Disease: A Systematic Review and Meta-analysis. JAMA Neurol. 2019 May 13;76(8):906-14. https://doi.org/10.1001/jamaneurol.2019.1120

$\square$ Cardiologia Croatica 2021;16(1-2):68. 